\title{
过渡金属催化氧气氧插入反应研究进展
}

\author{
吴锦雯朱雯李慧吴春雷沈润溥* 余乐茂*
}

(绍兴文理学院化学化工学院 浙江绍兴 312000)

\begin{abstract}
摘要 含氧结构骨架普遍存在于天然产物和生物活性小分子中，同时在有机合成中是一类重要的合成子，因此，含氧 化合物的合成一直是科学研究的热点. 过渡金属催化氧气氧插入反应策略是一类新颖而又高效构建碳碳键和碳杂键的 方法. 随着该类方法的应用和发展，它已成为含氧杂原子化合物最重要的合成方法之一. 在前人的工作基础上，同时 结合本课题组之前的工作，探讨了氧气环境条件下过渡金属催化醛酮类、烯炔烃、芳烃类及杂环芳烃类等参与的氧插 入反应及其在合成醇类、酯类、酰胺类和含氧杂环类等领域的新应用; 综述了基于 $\mathrm{C}-\mathrm{H}$ 键活化以及 $\mathrm{C}-\mathrm{C}$ 键断裂等 方式参与过渡金属催化氧气氧插入反应过程的研究新进展. 过渡金属催化剂在氧气氧化下，可以引发氧自由基历程的 氧插入反应，并实现氧自由基的高效和高选择性插入. 该类反应具有条件温和、操作简单、绿色环保和高原子经济性 等特点.
\end{abstract}

关键词 过渡金属催化; 氧气氧化; $\mathrm{C}-\mathrm{H}$ 活化; $\mathrm{C}-\mathrm{C}$ 断裂; 氧插入反应; 自由基历程

\section{Recent Advances of Transition Metal-Catalyzed Aerobic Oxygenation with Molecular Oxygen}

\author{
Wu, Jinwen Zhu, Jiawen Li, Hui Wu, Chunlei Shen, Runpu* Yu, Lemao* \\ (College of Chemistry and Chemical Engineering, Shaoxing University, Shaoxing, Zhejiang 312000)
}

\begin{abstract}
Oxygen-bearing structures are attractive synthetic targets due to their wide presence in a great number of natural products and biologically active molecules, and their role as useful synthons in organic synthesis. Thus, the preparation of O-bearing compounds has been an important topic. Transition metal-catalyzed aerobic oxygenation strategy has emerged as a novel and efficient methodology for constructing $\mathrm{C}-\mathrm{C}$ bonds and $\mathrm{C}$-heteroatom bonds. This strategy has become one of the most important and useful synthetic method in organic synthesis of O-bearing compounds. Based on the previous work of our group and others, herein, the new applications of transition metal-catalyzed oxygenation of $\mathrm{C}-\mathrm{H}$ bond activation and $\mathrm{C}-\mathrm{C}$ bond cleavage with oxygen as oxygen source are discussed. The recent progress in trans-metal catalytic oxygen insertion of ketones, aldehydes, alkene, alkynes, arene and aromatic heterocyclic compounds has been reviewed. Transition metal-catalysts can initiate oxygen insertion reaction of free radical pathway and realize selective insertion of oxygen radicals under oxygen oxidation. These reactions are characterized by mild conditions, environmental protection and highly atom economy.

Keywords transition metal catalysis; oxygen oxidation; $\mathrm{C}-\mathrm{H}$ activation; $\mathrm{C}-\mathrm{C}$ cleavage; oxygenated reaction; radical pathway
\end{abstract}

含氧化合物普遍存在于天然产物和生物活性分子 中，也是有机合成中重要的合成原料. 因此，含氧化合 物的合成和制备已经成为当前有机合成研究的热点，同 时受到了有机合成化学家们的广泛关注. 近十几年来, 很多新型合成含氧化合物的催化方法相继被报道 ${ }^{[1 \sim 3]}$. 其中, 过渡金属催化氧气氧插入反应是一类新型而又高
效构建 $\mathrm{C}-\mathrm{C}$ 键和 $\mathrm{C}-\mathrm{X}(\mathrm{X}=\mathrm{O}, \mathrm{N}$ 等 $)$ 的方 法 $^{[4]}$. 该类 反应在过渡金属催化下，可以实现氧原子的高选择性插 入, 从而高效地合成含氧杂原子的化合物. 同时, 由于 氧气的廉价易得及环境友好的特性，使得该类反应具有 操作简便、原子经济、绿色环保、无需隔绝空气和水等 特点. 该类反应的高效、绿色构建含氧化合物的优点使

\footnotetext{
* Corresponding authors. E-mail: yulm@usx.edu.cn; srunpu@usx.edu.cn

Received July 3, 2019; revised August 11, 2019; published online August 30, 2019.

Project supported by the Public Projects of Zhejiang Province of China (No. LGG19B02002) and the Doctoral Research Initiation Fund of Shaoxing University (No. 20185018).

浙江省公益技术项目(No. LGG19B02002)和绍兴文理学院博士科研启动基金(No. 20185018)资助项目.
} 
得过渡金属催化氧插入反应得以迅速发展, 尤其是铜催 化和钯催化的氧插入反应研究得到了极大的发展. 该类 反应在合成含氧化合物的应用也非常广泛, 研究对象主 要包括基本的酮类、醛类、芳烃类和炔烃类等, 涉及到 的反应类型包括羟基化反应、羰基化反应、酯化反应和 成环反应等. 对该类反应机理研究显示反应主要通过活 化 $\mathrm{C}-\mathrm{H}$ 键和 $\mathrm{C}-\mathrm{C}$ 键断裂两种基本方式实现氧气的插 入过程. 本文在前人研究报告的基础 上 $^{[5]}$, 同时结合本 课题组的工作, 不仅综述了过渡金属催化氧插入反应在 醛酮和烯炔烃的研究新进展, 同时还综述了该反应在去 芳构化和构建吲哚类衍生物等领域上的新应用.

\section{$1 \mathrm{C}-\mathrm{H}$ 键参与的氧插入反应}

过渡金属催化氧化 $\mathrm{C}-\mathrm{H}$ 键的氧插入反应主要包括 $\mathrm{C}\left(\mathrm{sp}^{3}\right)-\mathrm{H}$ 键、 $\mathrm{C}\left(\mathrm{sp}^{2}\right)-\mathrm{H}$ 键和 $\mathrm{C}(\mathrm{sp})-\mathrm{H}$ 键参与的氧插 入反应, 涉及到的研究对象包括烷烃类、烯烃类、芳烃 类和炔烃类等. 在过渡金属的催化氧化下, 氧气容易与 金属结合形成金属-氧自由基复合物，从而进行自由基 历程的 $\mathrm{C}-\mathrm{H}$ 键参与的氧插入反应, 实现 $\mathrm{C}-\mathrm{H}$ 键向 $\mathrm{C}-\mathrm{O}$ 键的转化.

\section{$1.1 \mathrm{C}\left(\mathrm{sp}^{3}\right)-\mathrm{H}$ 键参与的氧插入反应}

过渡金属催化氧化 $\mathrm{C}\left(\mathrm{sp}^{3}\right)-\mathrm{H}$ 键的氧插入反应可以 将甲基、亚甲基或次甲基转换成含羰基类或含氧杂环类 等化合物. 2011 年, Chiba 课题组 ${ }^{[6]}$ 通过以苄基苯甲腈(1) 为原料, 以分子内的 $\mathrm{N}-\mathrm{H}$ 亚胺基团导向经 1,5-氢迁移 的过程, 实现了铜催化茮位亚甲基 $\mathrm{C}\left(\mathrm{sp}^{3}\right)-\mathrm{H}$ 键参与的 氧插入反应, 最后通过酸化水解可以高效地得到了邻苯 二甲酰基取代的芳香类化合物 $\mathbf{3}$ (Scheme 1).

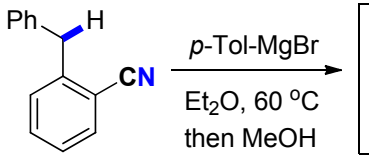

1

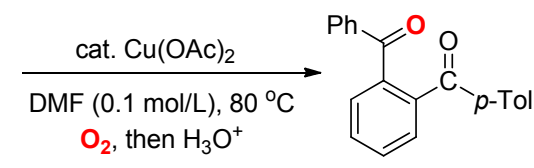

3, up to $91 \%$ yield
图式 1 亚胺导向苄基参与的氧插入反应

Scheme 1 Imine-directed benzyl-participated oxygenation

2012 年, Chiba 课题组 ${ }^{[7]}$ 通过溴化亚铜-双吡啶体系 催化, 采用同样的亚胺基团导向和 1,5-氢迁移等策略进 一步实现了次甲基参与的铜催化氧插入反应, 一步构建 了含二氢噁唑环结构的化合物 5 (Eq. 1). 反应机理研究 显示反应先生成叔碳自由基中间体, 随后进行铜氧条件
下的氧插入反应过程, 最后环化水解得目标产物.

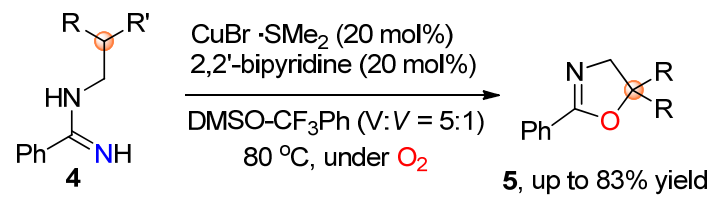

2011 年, 焦宁课题组 ${ }^{[8]}$ 通过澳化亚铜-吡啶催化芳 基乙酫与苯胺之间的氧插入反应，实现了对 $\alpha$-芳基酮酰 胺类化合物 8 的一步构建(Eq. 2). 相比于传统合成 $\alpha$-芳基 酮酰胺类化合物方法, 该反应过程涉及到两个 $\mathrm{C}\left(\mathrm{sp}^{3}\right)-\mathrm{H}$ 键、一个 $\mathrm{C}\left(\mathrm{sp}^{2}\right)-\mathrm{H}$ 键、一个 $\mathrm{N}-\mathrm{H}$ 键和 $\mathrm{O}-\mathrm{O}$ 键的断 裂, 同时表现出很好的底物适用性范围. 反应机理研究 显示底物芳基乙醛与苯胺首先进行脱水缩合反应生成 含亚胺结构的中间体，随后进行过渡金属催化的氧插入 反应.

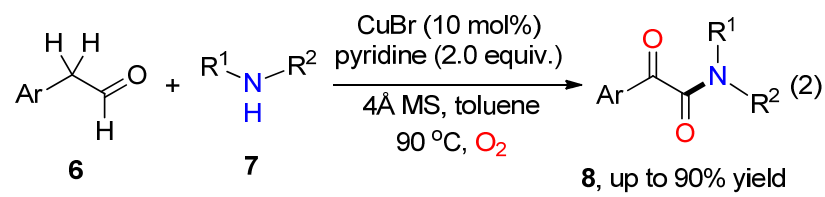

2012 年, 焦宁课题组 ${ }^{[9]}$ 进一步通过溴化亚铜一吡啶 催化芳基乙醛和苯胺之间的氧插入反应, 在不加入分子 篮和降低温度的条件下，实现了对 2-乙酰基榺类化合物 10 的合成. 反应机理研究显示, 反应过程经过氨基自由 基正离子加成反应，生成含脒结构的自由基中间体; 随 后经过活化 $\mathrm{C}\left(\mathrm{sp}^{3}\right)-\mathrm{H}$ 键的氧插入反应. 整个反应过程 脱去了 6 个氢(Eq. 3).

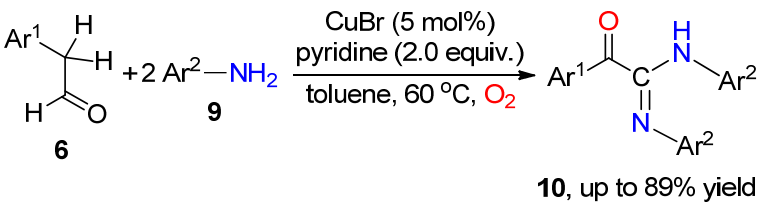

同年, 焦宁课题组 ${ }^{[10]}$ 以具有 $\alpha-\mathrm{H}$ 的伯胺和芳基乙醛 为原料, 在氧气的环境中, 进一步通过澳化铜一吡啶催 化体系催化的氧插入反应，一步合成了 2,4-双取代的噁 唑类化合物 12 (Eq. 4). 该反应涉及到六个氢原子的消 除. 相比传统合成啞唑类的方法, 该反应的底物廉价易 得，更适合于工业应用.

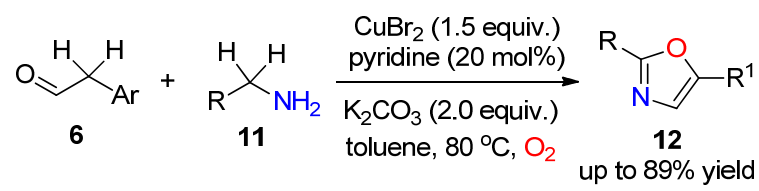

2015 年, 焦宁课题组 ${ }^{[11]}$ 通过溴化亚铜-哌啶体系催 化芳基乙酮与伯醇之间氧插入反应，实现了一价铜催化 甲基参与的氧插入反应。该反应过程通过哌啶活化 
$\mathrm{C}(\alpha)-\mathrm{H}$ 键, 氧化氧气插入生成 $\alpha$-酮醛中间体. 该中间 体随后与伯醇进行亲核加成反应, 最终氧化脱氢得 $\alpha$-酮 酸酯类化合物 15 (Eq. 5).

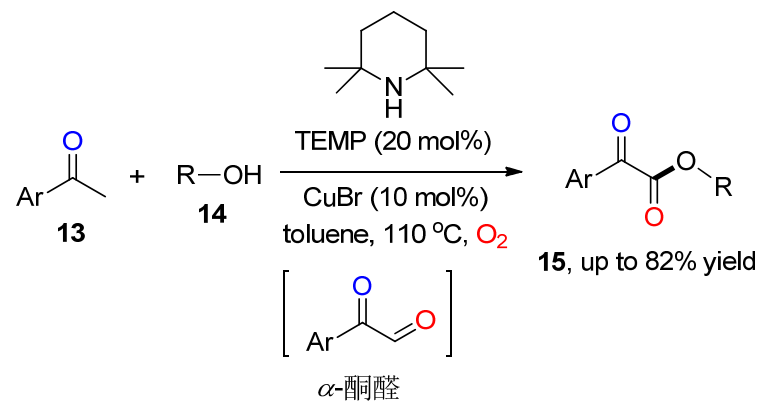

2016 年, Kumar 课题组 ${ }^{[12]}$ 通过二价铜一吡啶体系催 化苄位氢的氧化氧插入反应, 首次实现了从单底物的芳 基亚胺酸酯向芳基 $\alpha$-酮酸酯的转化. 该反应方法具有操 作简单及底物范围广等特点. 同时, 研究该反应机理时 显示产物中其中一个氧原子来自反应中的水(Eq. 6).

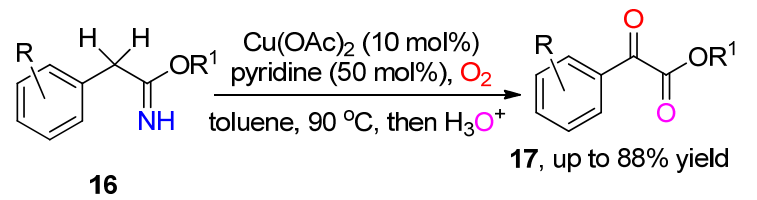

过渡金属催化活化 $\mathrm{C}\left(\mathrm{sp}^{3}\right)-\mathrm{H}$ 键参与的氧插入反应 还可以被用于合成芳酰伯胺类化合物. 2017 年, 宋秋玲 课题组 ${ }^{[13]}$ 发展了一条以苯乙腈和尿素为底物, 二氯化 亚铁催化活化苄位 $\mathrm{C}\left(\mathrm{sp}^{3}\right)-\mathrm{H}$ 键的氧插入反应, 实现了 对芳基甲酰胺类化合物的合成, 同时伴随着良好的产率 (Scheme 2). 反应机理研究显示尿素为氨基的来源. 同 年, 孙绍发课题组 ${ }^{[14]}$ 通过氧化亚铜催化，在强碱条件下 实现了苯乙腈与氯化铵之间的氧插入反应, 合成了芳酰 伯胺类化合物(Scheme 2). ${ }^{18} \mathrm{O}$ 同位素标记实验显示产物 中的氧全部来自于氧气.

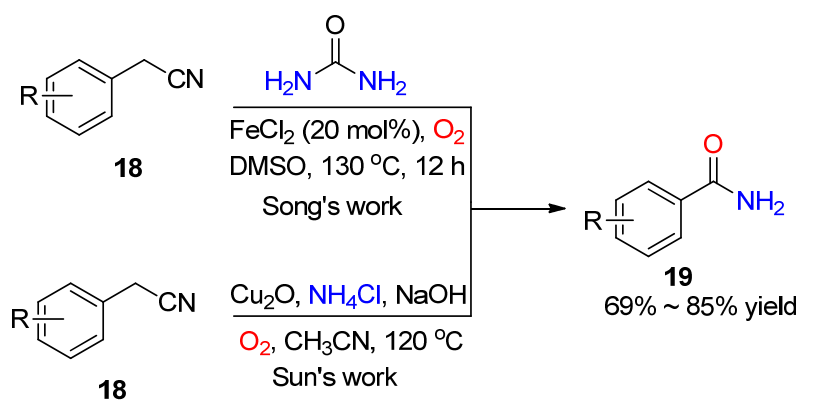

图式 2 铜/铁催化芳基乙腈的氧插入反应 Scheme 2 Copper/iron catalyzed oxygenation of aryl acetonitrile

黄德光课题组 ${ }^{[15]}$ 利用三齿配体的双吡啶类化合物 与三氟甲磺酸铜配位, 得到金属复合物. 该金属复合物
在强碱叔丁醇钾的作用下，可以催化室温下的 2-吡啶甲 基芳胺为底物的氧插入反应，一步构建了 $N$-苯基-2-吡 啶甲酰胺类化合物 21 (Eq. 7). 该反应条件温和是配体 辅助催化的一大特点, 然而, 该方法的产物专一性较差, 同时会伴随着吡啶酸和亚胺等副产物的生成.

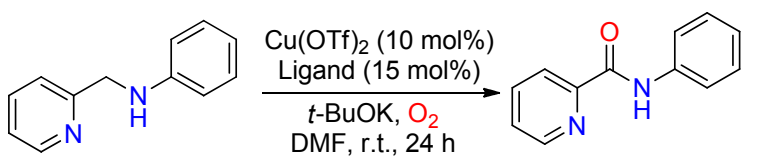

20

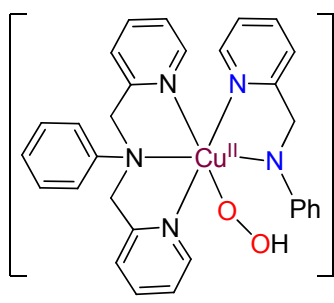

21, up to $92 \%$ yield

\section{$1.2 \mathrm{C}\left(\mathrm{sp}^{2}\right)-\mathrm{H}$ 键或 $\mathrm{C}=\mathrm{C}$ 键参与的氧插入反应}

过渡金属催化氧化 $\mathrm{C}\left(\mathrm{sp}^{2}\right)-\mathrm{H}$ 键的氧插入反应主要 包括端烯、内部烯烃和芳环上 $\mathrm{C}-\mathrm{H}$ 键参与的氧插入反 应. 该类反应通常先经过自由基的加成反应，生成新的 自由基中间体，随后进行 $\mathrm{C}\left(\mathrm{sp}^{3}\right)-\mathrm{H}$ 的氧气插入反应. 2013 年, 焦宁课题组 ${ }^{[16]}$ 通过三氟甲磺酸铜和硝酸铁共 同催化，实现了芳基肼与取代的苯乙烯之间的氧插入反 应，一步合成了 1,2-二芳基取代的乙二酮类化合物 24 . 进一步的反应底物拓展结果显示, 该反应适用范围较 窄，仅限于端烯类化合物(Eq. 8).

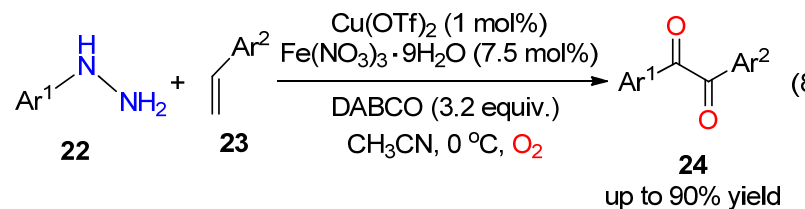

2015 年，焦宁课题组 ${ }^{[17]}$ 通过过渡金属锰催化烯烃 与三甲基叠氮硅烷之间的氧插入反应，实现了对 $\beta$-叠氮 醇类化合物 27 的高效合成. 该反应不仅可以在室温条 件下进行，而且具有很好的底物适用性，端烯和内烯都 可以参与反应. 密度泛函理论(DFT)结果显示, $\beta$-叠氮取 代的自由基中间体和三价锰中间体的生成是该反应成 功转化的关键步骤(Scheme 3).

同年，雷爱文课题组 ${ }^{[18]}$ 通过氯化亚铜和醋酸钴分别催 化异着肟酸酯与端烯和 1,1-二取代端烯之间的氧插入反 应，实现了对 $\alpha$-氧基酮 30 和 $\alpha$-氧三级醇类化合物 31 的 合成. 该方法具有较高的化学选择性且不需要任何配体 或碱等辅助添加剂等特点. 反应的机理研究显示反应先 生成异羟肟自由基，随后进行自由基的加成反应，最后 实现氧自由基插入反应(Scheme 4). 


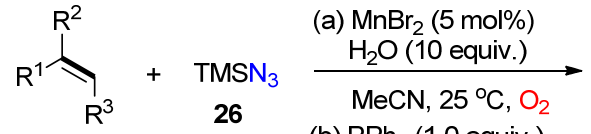

25 (b) $\mathrm{PPh}_{3}$ (1.0 equiv.)
27, up to $94 \%$ yield

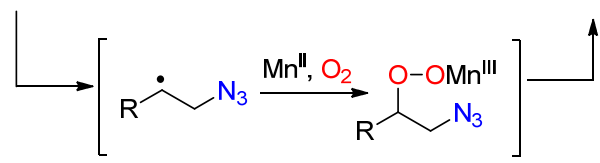

图式 3 锰催化氧插入反应合成 $\beta$-叠氮醇类化合物

Scheme 3 Synthesis of $\beta$-azidol compounds by $\mathrm{Mn}^{2+}$ catalyzed oxygenation

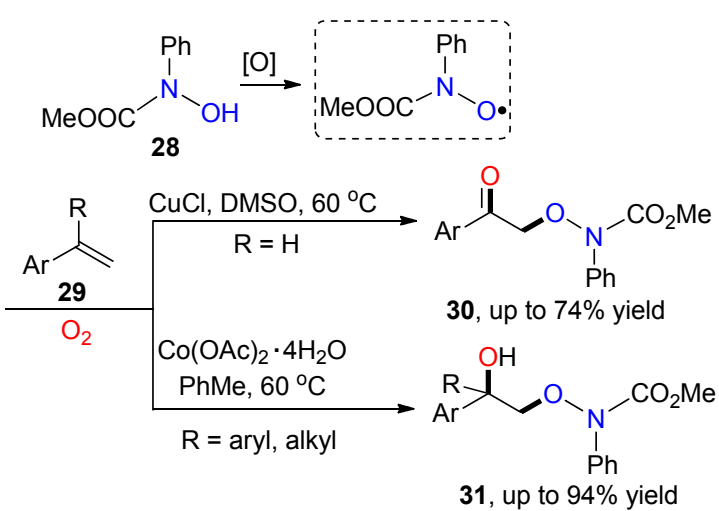

图式 4 铜/钴催化氧肟酸与端烯之间的氧插入反应 Scheme 4 Copper/cobalt catalyzed oxygen insertion reaction between oxime acid and terpene

砜类化合物具有广泛的生物或药物活性，一直是有 机合成化学家的研究内容之一 ${ }^{[19]} .2013$ 年, 王华课题 组 ${ }^{[20]}$ 通过醋酸酮催化, 氧气活化内烯烃与磺酰胇之间 的氧插入反应, 实现了对 $\beta$-羰基砜类化合物 33 的合成. 机理研究显示反应首先经过磺酰肼的氧化, 生成磺酰自 由基中间体. 2014 年, 王桦课题组 ${ }^{[21]}$ 进一步通过四水氯 化亚铁催化的亚磺酸与内烯烃之间的氧插入反应, 实现 了对 $\beta$-羰基砜类化合物的合成, 产率良好(Scheme 5).

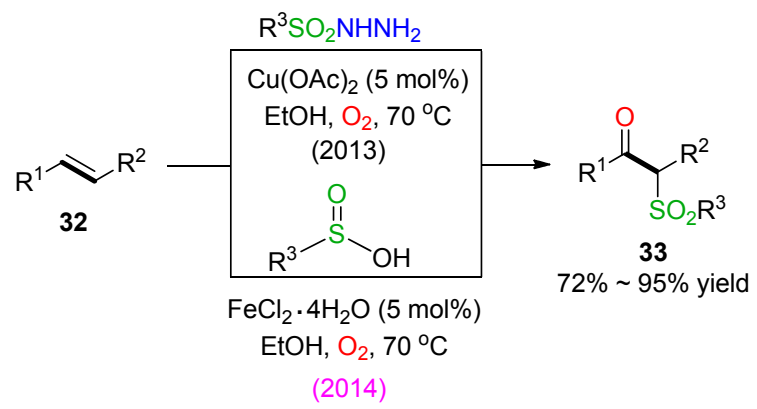

图式 5 铜/铁催化的氧插入反应合成 $\beta$-羰基砜类化合物

Scheme 5 Copper/iron catalyzed oxygenation for the synthesis of $\beta$-carbonyl sulfones

2018 年, 魏文龙课题组 ${ }^{[2]}$ 通过五水硫酸铜催化氧 气氧化, 实现了芳基端烯、磺酸纳盐、芳基重氮盐及氧
气等四组分之间的氧插入反应，一锅法合成了 $\alpha$-芳肼 基- $\beta$-酮砜类化合物 36. 同时，该反应方法表现出很好的 底物适用性. 反应的机理研究显示反应过程首先经过砜 自由基的加成，随后进行氧气插入反应. 该反应同时涉 及到对 $\mathrm{C}-\mathrm{O}$ 键、 $\mathrm{C}-\mathrm{S}$ 键和 $\mathrm{C}-\mathrm{N}$ 键一锅法的构建( $\mathrm{Eq}$. 9).

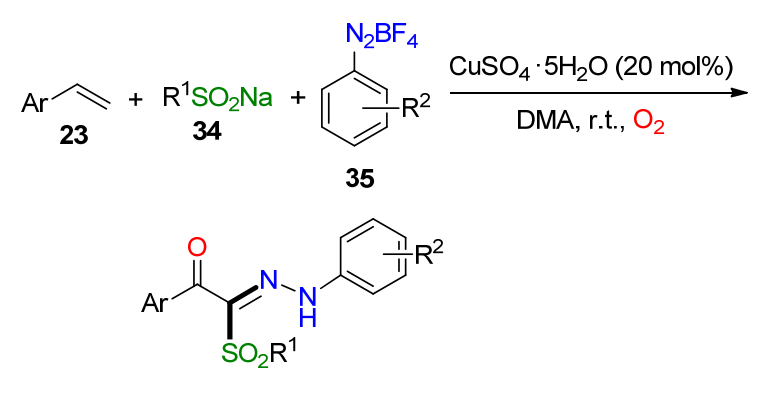

36. up to $85 \%$ yield

利用分子内活性基团与活性基团之间的反应是合 成环状化合物常用的方法. 而利用分子内环化反应也可 以实现分子参与的环化-插氧串联反应. 2011 年, Chiba 课题组 ${ }^{[23]}$ 通过二价的醋酸铜催化实现了分子内的环化 氧插入反应. 该反应以同时含端烯基团与缺电子烯胺基 团的仲胺类化合物为底物, 在铜氧催化体系下, 一步构 建了二取代 3-吡咯甲醛类化合物 38, 伴随着良好的产 率和底物适用性(Eq. 10).

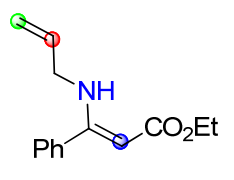

37
38, up to $83 \%$ yield

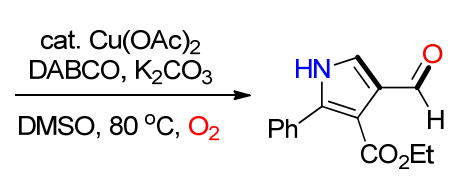

过渡金属催化芳基参与的氧插入反应主要通过活 化芳环上的 $\mathrm{C}\left(\mathrm{sp}^{2}\right)-\mathrm{H}$ 键实现氧的插入. 该部分涉及到 的对象为芳烃类和杂环芳烃类，涉及到的反应包括 $\mathrm{C}\left(\mathrm{sp}^{2}\right)-\mathrm{H}$ 键的羟基化反应和芳基参与的去芳构化反 应 ${ }^{[24]} .2009$ 年, 余金权教授课题组 ${ }^{[25]}$ 通过醋酸钯催化活 化苯环上的 $\mathrm{C}\left(\mathrm{sp}^{2}\right)-\mathrm{H}$ 键, 实现了苯甲酸类化合物的氧 插入反应, 合成了邻羟基苯甲酸类化合物(Eq. 11). 反应 机理研究显示产物中的氧来自于氧气, 同时该反应表现 出很好的底物适用性.

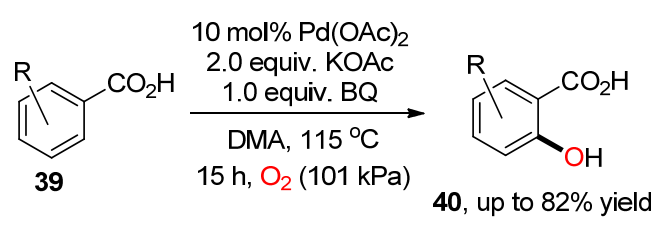

无独有偶, 2013 年, 焦宁课题组 ${ }^{[26]}$ 通过二价的氯化 钯催化、 $N$-羊基茎苯邻二甲酰胺辅助催化氧化 $\mathrm{C}\left(\mathrm{sp}^{2}\right)-\mathrm{H}$ 
键，实现了对 2-芳基吡啶环的 $\mathrm{C}-\mathrm{H}$ 键的羟基化反应，并 伴随着良好的产率. 反应机理研究显示, 底物吡啶环与 钯配位，生成双配位的金属络合物中间体，在氮杂原子 的孤对电子诱导效应下活化苯环上邻位 $\mathrm{C}-\mathrm{H}$ 键(Eq. 12).

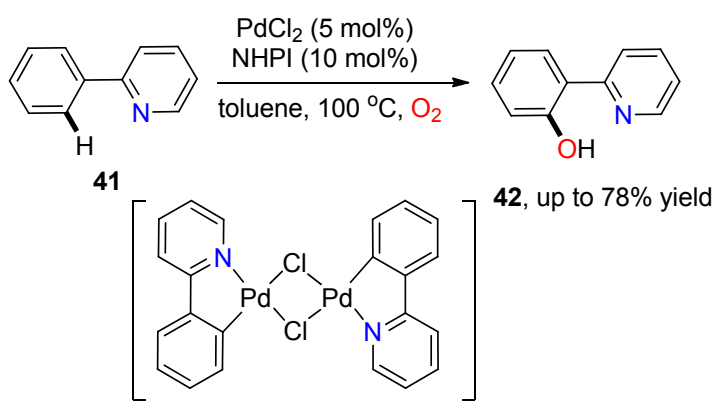

2010 年, Chiba 等 ${ }^{[27]}$ 以 $\alpha$-叠氮- $N$-苯基酰胺为底物, 发展了一条由二价铜催化的分子内环化-氧插入反应, 合成了一系列螺环醌类化合物, 实现了对苯环的去芳构 化. 推测的反应机理显示, 反应涉及到苯胺环上对位 $\mathrm{C}\left(\mathrm{sp}^{2}\right)-\mathrm{H}$ 键的活化和氧插入过程(Eq. 13).

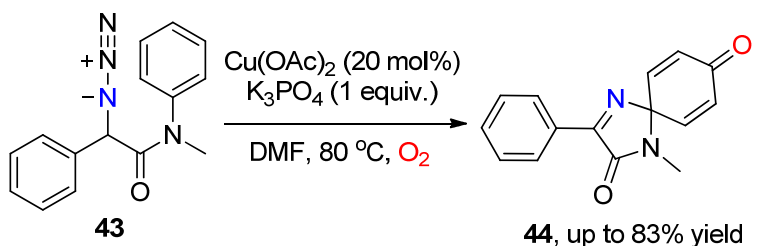

2012 年, Chiba 课题组 ${ }^{[28]}$ 又发展了一种基于亚胺中 间体活化苯环和萗环上 $\mathrm{C}\left(\mathrm{sp}^{2}\right)-\mathrm{H}$ 键的氧插入反应. 该 方法以 3-甲基-2 芳基苯甲腈为底物, 先与格氏试剂对甲 苯基溴化镁进行亲核加成反应生成亚胺中间体，随后铜 催化苯环和萘环的 1,4-氨基氧插入反应过程，一锅法实 现了对苯环和䒺环的氧插入和氨基插入去芳构化的螺 环化反应(Scheme 6).

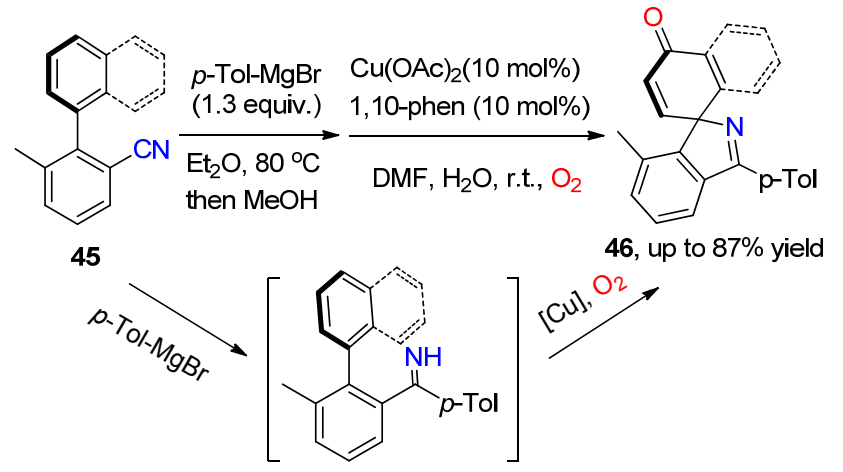

图式 6 铜催化芳环的氧插入螺环反应

Scheme 6 Copper catalyzed spiral cyclization of aromatic rings with oxygenation

2015 年, 焦宁课题组 ${ }^{[29]}$ 发展了一条钯催化氧气活
化苯环上 $\mathrm{C}-\mathrm{H}$ 硝基化和酰基化反应(Scheme 7). 该反 应以叔丁基亚硝酸盐(TBN)和甲苯作为自由基前体，通 过氧气作为氧化剂用于激发自由基前体，同时作为氧源 进行氧气插入. 对于苯环的酰基化反应, 加入自由基引 发剂 $N$-着基苯邻二甲酰胺辅助催化促使苯甲酰基自由 基的生成. 对于苯环硝基化反应，控制实验显示硝基中 的氧有可能来自于氧气，也有可能来自于底物本身

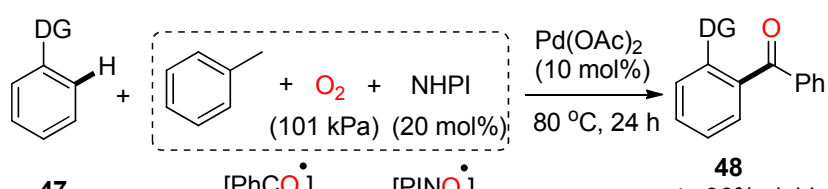

$47 \quad\left[\mathrm{PhCO}^{\circ}\right] \quad\left[\mathrm{PINO}{ }^{\circ}\right] \quad$ up to $96 \%$ yield

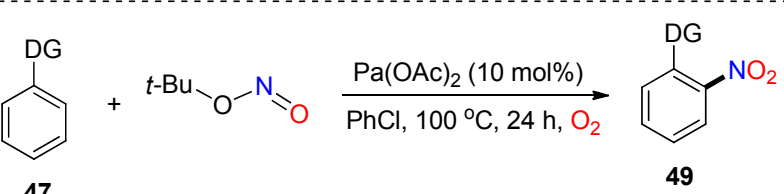

up to $81 \%$ yield

图式 7 钯催化氧插入的酰基化和硝基化反应

Scheme 7 Palladium-catalyzed acylation and nitrogenation of Aromatic rings with oxygenation

2015 年，焦宁课题组 ${ }^{[30]}$ 以芳基乙醛和芳基伯胺为 底物, 在氧气环境中, 以三氟乙酸铜为催化剂, 实现了 两者之间的氧插入反应，合成了 $N$-芳基取代吡啶酮类 化合物(Eq. 14). 反应机理推测可能先生成吡啶环，而后 经过活化脱茮基得吡啶正离子中间体，最后铜催化氧插 入得到目标产物 51. ${ }^{18} \mathrm{O}$ 同位素标记试验显示产物中的 氧同时来源于水或氧气.

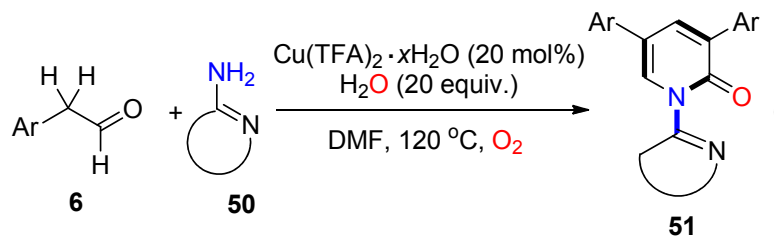

up to $93 \%$ yield

值得注意的是，除了苯环上的 $\mathrm{C}\left(\mathrm{sp}^{2}\right)-\mathrm{H}$ 参与的氧 插入反应, 利用吲哚参与的氧插入反应合成吲哚类化合 物也是有机合成研究的热点，近几年对吲哚参与的氧插 入反应也得到了迅速的发展. 由于吲哚结构的特点，其 3-位 $\mathrm{C}\left(\mathrm{sp}^{2}\right)-\mathrm{H}$ 键化学性质活泼, 易发生 $\mathrm{C}-\mathrm{H}$ 功能化反 应. 2011 年, 苏伟平课题组 ${ }^{[31]}$ 报道了通过醋酸钴和醋酸 锰共同催化, $N$-差基苯邻二甲酰胺辅助催化, 氧气氧化 的吲哚与 $\beta$-酩酸酯的氧化交叉偶联反应，得到了 3 -位酮 基化-2-烯化的吲哚酮类化合物 54. 与之类似的工作由 刘良先课题组 ${ }^{[32]}$ 报道，以 2,2,6,6-四甲基哌啶一氮一氧化 物(TEMPO)为氧化剂, 氧气条件下, 硫酸铜为催化剂, 实现了吲哚与乙酰乙酸乙酯之间的氧气插入反应，完成 
了对吲哚酮类化合物的合成(Scheme 8).

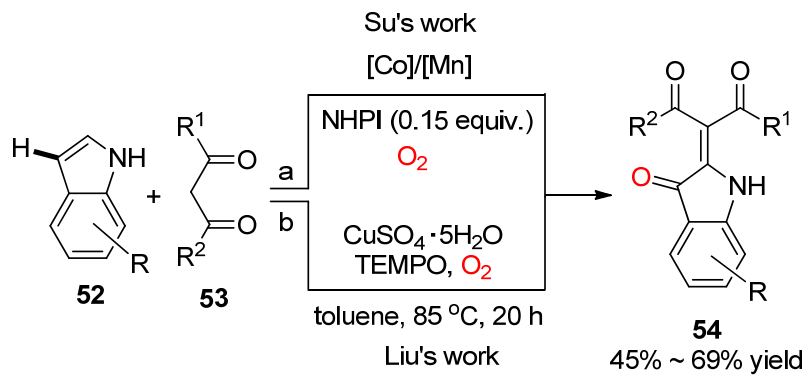

图式 8 过渡金属催化吲哚与 1, 3-二酮之间的氧插入反应 Scheme 8 Transition metal catalyzed oxygenation between indole and 1,3-diketone

2013 年, 王彦广课题组 ${ }^{[33]}$ 报道了在氧气环境中, 碘 化亚铜催化吲哚与靛红之间的氧插入环化反应, 实现了 对色胺酮类化合物的合成. 反应的机理研究显示吲哚在 铜氧体系催化条件下, 可以生成中间体靛红, 随后经过 脱羧基偶联, 分子内的亲核加成反应, 最后氧化芳构化 得到最终色胺酮类产物 56 (Scheme 9).

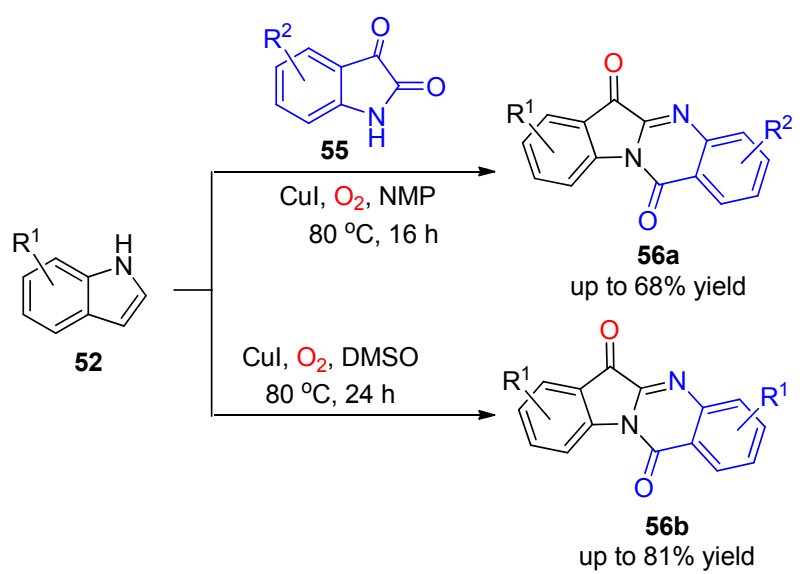

图式 9 铜氧催化氧插入反应合成色胺酮类化合物 Scheme 9 Copper catalyzed oxygenation for the synthesis of tryptamine compounds

2016 年, 邓国军课题组 ${ }^{[34]}$ 发展了一条由铜催化氧 气活化的吲哚 3-位 $\mathrm{C}-\mathrm{H}$ 参与的氧插入环化反应. 该反 应在氯化铜为催化剂的条件下, 通过氧气氧化 2-取代吲 哚与苯乙酮肜之间的氧气插入环化过程, 一锅法实现了 对吲哚的多功能化反应(Eq. 15). 反应的机理研究显示, 该反应通过一个自由基的反应机理，苯乙酮肟作为体内 氧化引发剂激发氧气氧化插入反应.

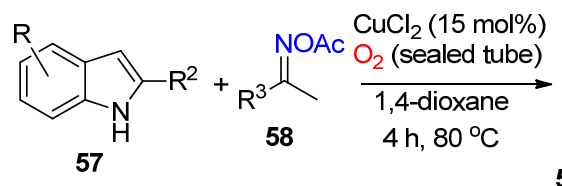<smiles>[R]C1=NN2c3ccccc3C(=O)C2([R])C1</smiles>
59 , up to $85 \%$ yield
2018 年, 范学森课题组 ${ }^{[35]}$ 发展了溴化亚铜催化邻 苯甲酰胺-2-取代吲哚的氧插入反应. 该反应在空气环 境中，以 $N, N$-二甲基甲酰胺(DMF)为溶剂，可以实现对 吲哚-3-酮类骨架的构建(Scheme 10). 此外, 该反应在 1,4-二氧六环为溶剂时, 生成的是吲哚并喹啉结构类化 合物。

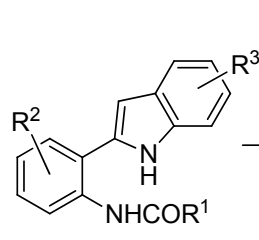

60

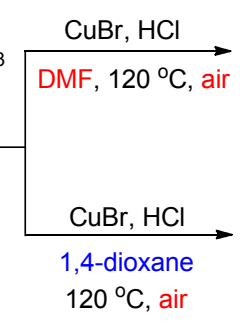<smiles>[R]C=Cc1ccccc1NC([R])=O</smiles>

61, up to $72 \%$ yield<smiles>[R]c1ccc2nc([R])n3c4ccccc4cc3c2c1</smiles>

图式 10 铜催化氧插入反应合成吲哚 3-酮类化合物 Scheme 10 Copper catalyzed oxygenation for the synthesis of indole 3-ketones

2019 年, Borban 等 ${ }^{[36]}$ 发展了一种由氯化亚铜-吡啶 体系催化的 $3 H$-吲哚与叔丁氧基差胺之间的氧插入反 应，实现了对 2-叔丁氧酰胺基取代的吲哚-3-酮类化合 物 64 的合成(Eq. 16). 然而, 该反应具有一定的底物局 限性. 在该条件下，当 3-取代吲哚参与反应时，并不能 生成吲哚酮类化合物，而是进一步实现对 2-位甲基的功 能化的产物.

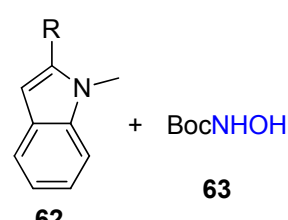

62

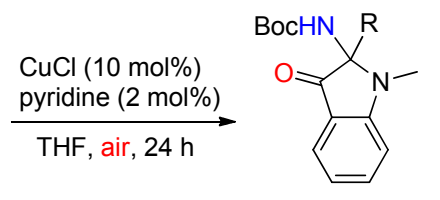

64

up to $77 \%$ yield
2018 年，本课题组 ${ }^{[37,38]}$ 发展了一条铜催化 3-取代吲 哚的碳碳双键的氧插入环化反应(Eq. 17). 在碱式碳酸 铜和醋酸铜的催化作用下, 通过氧化吲哚 $\mathrm{C}=\mathrm{C}$ 双键生 成双氧桥环结构的中间体，随后进一步氧化消去得到氧 杂三元环结构的二氢吲哚中间体. 该中间体通过与偶氮

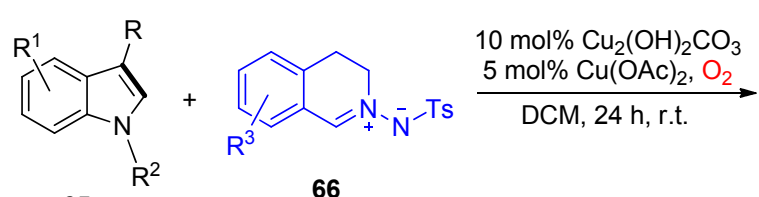

65

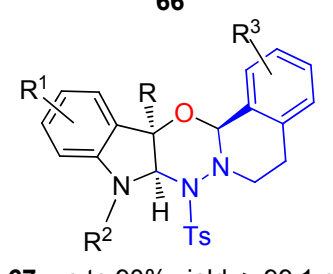


甲碱亚胺类的环化反应得到含氧杂环的二氢吲哚类衍 生物 67, 并且伴随着非常高的非立体选择性, 同时含有 多个手性中心. 对该反应的机理研究显示反应过程中的 空间位阻和 $\pi$ 电子作用是该反应具有高非立体选择性的 主要原因.

\section{$1.3 \mathrm{C}(\mathrm{sp})-\mathrm{H}$ 键或 $\mathrm{C} \equiv \mathrm{C}$ 键参与的氧插入反应}

过渡金属催化 $\mathrm{C}(\mathrm{sp})-\mathrm{H}$ 键参与的氧插入反应主要 研究对象为端炔烃类. 与烯烃类参与的氧插入反应类 似, 炔烃首先进行金属催化的加成反应, 而后进行氧化 活化 $\mathrm{C}\left(\mathrm{sp}^{2}\right)-\mathrm{H}$ 或 $\mathrm{C}\left(\mathrm{sp}^{3}\right)-\mathrm{H}$ 键的氧气插入反应过程. 2010 年, 焦宁课题组 ${ }^{[39]}$ 通过溴化亚铜-吡啶体系催化, 加入自由基捕捉剂四甲基哌啶氧化物(TEMPO)辅助催 化，实现了芳基伯胺与炔烃之间的氧插入反应，合成了 $\alpha$-酮酰胺类化合物 69. 可能的反应机理显示该反应过程 先经过脱质子氨基化反应过程, 得到烯胺类中间体, 然 后经 $\mathrm{C}\left(\mathrm{sp}^{2}\right)-\mathrm{H}$ 键的氧插入反应得到关键过氧四元环氮 自由基中间体. ${ }^{18} \mathrm{O}$ 标记实验显示产物中的氧全部来自 于氧气, 从而显示反应过程需要断裂 $\mathrm{O}-\mathrm{O}$ 键(Eq. 18).

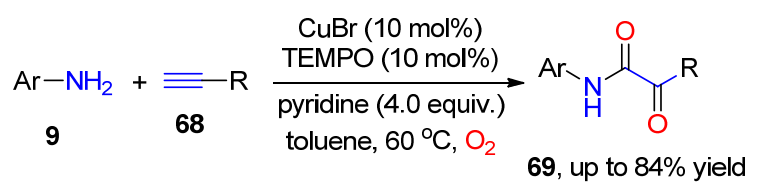

2011 年, 王彦广课题组 ${ }^{[40]}$ 报道了以氧气为氧源, 溴 化亚铜催化炔烃、磺酰叠氮和 $3 H$-吲哚三组分之间的氧 插入反应, 合成了 3-位含羰基的吲哚类化合物 72. 反应 机理的研究显示, 反应过程首先经叠氮与炔烃在铜催化 下反应原位形成的烯亚胺过渡态, 随后与吲哚发生亲电 加成反应得到关键的烯胺中间体. 最后, 经过铜氧催化 氧化 $\mathrm{C}\left(\mathrm{sp}^{2}\right)-\mathrm{H}$ 键的氧插入反应得到最终产物(Eq. 19).

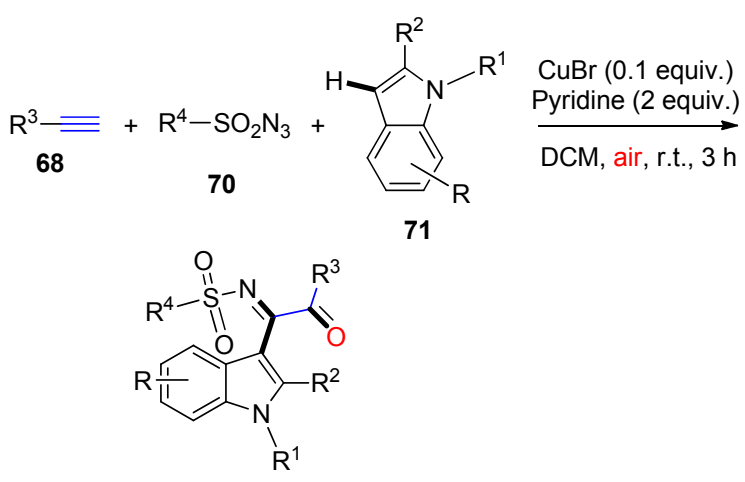

72, up to $80 \%$ yield

内部炔烃也可以作为底物实现过渡金属催化的氧 插入反应, 反应过程不仅仅只涉及到对 $\mathrm{C}\left(\mathrm{sp}^{3}\right)-\mathrm{H}$ 键的 氧化. 继分子内烯烃参与的氧插入反应之后, Chiba 课题 组 ${ }^{[41]}$ 在 2012 年进一步通过溴化亚铜和 1,10 -邻二氮杂菲
联合催化，实现了内炔与烯胺基团之间的分子内环化氧插入反应. 该反应首先通过活化烯胺的 $\mathrm{C}\left(\mathrm{sp}^{2}\right)-\mathrm{H}$ 键 与炔基进行分子内环化反应和电子重排，生成苠自由基 中间体; 再经过 $\mathrm{C}\left(\mathrm{sp}^{3}\right)-\mathrm{H}$ 键参与铜催化氧化氧气插入 过程，高效合成了芳酰基取代的喹啉类化合物 74 (Scheme 11). 在该反应条件中, 通过二价的醋酸铜催化, 进一步实现了内炔与亚胺之间的氧插入反应，伴随着对 含羰基取代的咪唑和嘧啶类衍生物 76 的合成(Scheme 11).
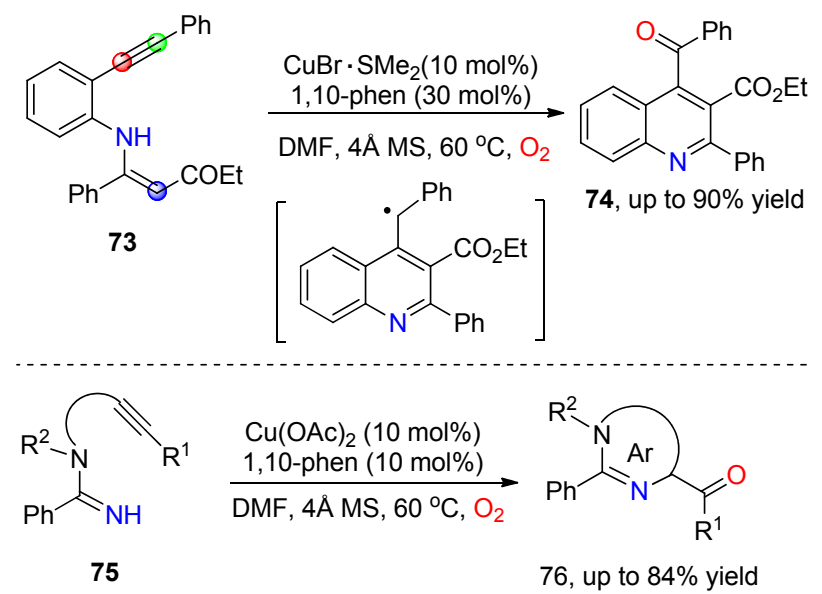

图式 11 铜催化炔烃参与的分子内环化-氧插入反应 Scheme 11 Copper catalyzed intramolecular cyclizationoxygenation involved by internal alkynes

2014 年，马成课题组 ${ }^{[42]}$ 通过氯化钯和溴化铜联合 催化以烯二炔烃-酰亚胺类或羧酸类化合物 77 和内部炔 烃为底物的氧插入反应，经过串联的偶联一铜催化氧插 入反应过程, 实现了对异吲哚啉酮类结构或邻酰基苯甲 酸类化合物 80 的构建(Scheme 12). 整个反应过程涉及 到一个 $\mathrm{C}\left(\mathrm{sp}^{2}\right)-\mathrm{H}$ 键的断裂. 邻酰基苯甲酸类结构可以 进一步经过脱羧反应得到 1,4-二酰基苯类化合物. 该反 应的机理研究显示反应通过[4+2]组合方式实现产物的 芳构化. 2016 年，马成课题组 ${ }^{[43]}$ 在前期工作的基础上进 一步通过过渡金属盐氯化钯和碘化亚铜联合催化，在氧 气环境中, 实现了烯二炔类底物自身的二聚一氧插入串 联反应，高效地合成了二酰基取代的萗甲酸类化合物 81 (Scheme 12). 同位素标记实验显示产物中新加入的 氧原子分别来自于水和氧气. 值得一提的是，该类反应 具有非常高的原子经济性，没有任何的副产物产生.

2017 年，马成等 ${ }^{[44]}$ 通过二价的钯/铜联合催化体系, 以类似的交叉环化-氧插入串联反应方式, 进一步实现了 烯二炔与炔基苯胺类化合物之间的氧插入反应，高效地 合成了酰基取代的菲啶类衍生物 83. 控制实验显示反应 为自由基反应历程, 且产物新生成羰基中的氧全部来自 于氧气. 反应机理研究显示, 反应涉及到叔碳自由基中 间体的生成及 $\mathrm{C}\left(\mathrm{sp}^{3}\right)-\mathrm{H}$ 的氧插入反应过程(Eq. 20). 
<smiles>[R]C#CC(=O)C(=O)NC1CCCCC1</smiles>

$(\mathrm{X}=\mathrm{NTS}$ or $\mathrm{O})$

77

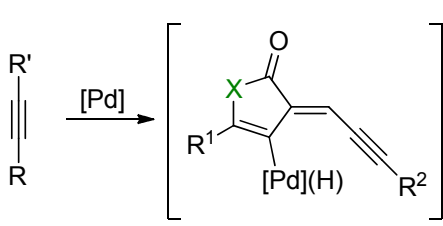

79

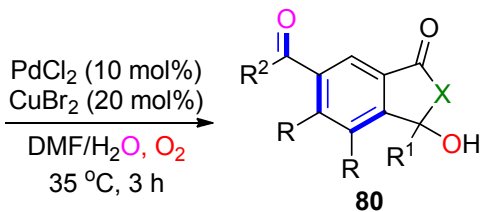

up to $95 \%$ yield

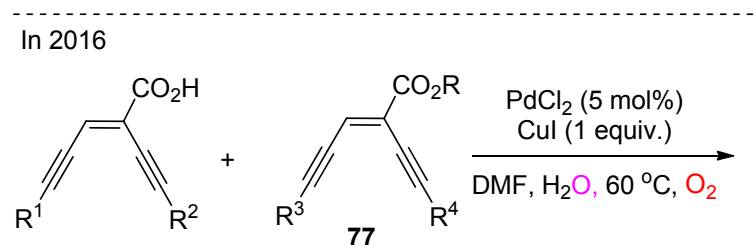<smiles>[R]C(=O)c1cc2c([R])c(C([R1])=O)c(C(=O)O)cc2c([R])c1C([R])=O</smiles>

81

up to $92 \%$ yield

图式 12 钯/铜催化的炔烃之间的偶联-氧插入串联反应 Scheme 12 Palladium/copper catalyzed coupling-oxygenation cascade between alkynes

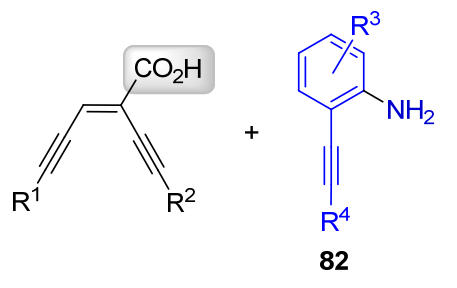

$\mathrm{PdCl}_{2}(5 \mathrm{~mol} \%)$ $\mathrm{CuBr}_{2}$ (0.2 equiv.), $\mathrm{O}_{2}$ $3 \AA \mathrm{MS}, 30^{\circ} \mathrm{C}, \mathrm{DMF}$ then $\mathrm{CH}_{3}, \mathrm{~K}_{2} \mathrm{CO}_{3} 40^{\circ} \mathrm{C}$<smiles>[R]C(=O)c1c(C(C)=O)cc2c([R])nc3c[R1]ccc3c2c1[R4]</smiles>

83, up to $91 \%$ yield

2018 年, 焦宁课题组 ${ }^{[45]}$ 进一步以 $N$-碘代琥珀酰亚 胺(NIS) 作为辅助添加剂, 通过溴化铜一吡啶催化体系催 化, 实现了芳基伯胺与吸电子取代的炔烃之间的氧化插 入反应，一步合成了 2,4,5-三取代的噁唑类化合物 86 (Eq. 21). 反应过程中会生成烯胺中间体，同时涉及到一 个 $\mathrm{C}\left(\mathrm{sp}^{2}\right)-\mathrm{H}$ 键的断裂. 对照试验结果显示, $N$-碘代琥珀 酰亚胺可以促进不饱和碳氢键断裂, 表现出辅助消去氢 自由基的作用.

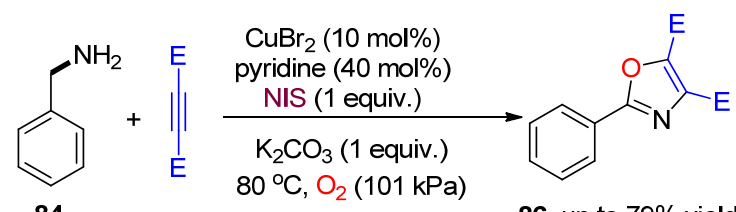

84
85 toluene/<smiles>FC(F)=CCc1ccccc1</smiles>

烯胺中间体

通过过渡金属催化氧化 $\mathrm{C}-\mathrm{H}$ 键的氧插入反应涉及 到氢原子的消除，所以通常会伴随着水分子或氨气等副 产物的产生. $\mathrm{C}-\mathrm{H}$ 键参与的氧插入反应应用范围广泛, 可以构建不同类型的醇酚类、酮酯类、酰胺类、含氧杂 环类以及吲哚酮类化合物. 除了活化 $\mathrm{C}-\mathrm{H}$ 键之外, 过 渡金属还可以通过催化断裂 $\mathrm{C}-\mathrm{C}$ 键的方式进行氧气插 入反应.

\section{2 断裂 $\mathrm{C}-\mathrm{C}$ 键的氧插入反应}

过渡金属催化断裂 $\mathrm{C}-\mathrm{C}$ 键的氧插入反应过程中往 往会生产过氧环中间体，涉及到碳碳键和氧氧键的同时 断裂. 2012 年, 焦宁课题组 ${ }^{[46]}$ 进一步以二水醋酸锰催化, 通过断裂 $\mathrm{C}-\mathrm{C}$ 键和 $\mathrm{O}-\mathrm{O}$ 键的反应过程, 实现了醛与 仲胺之间的氧插入反应，合成了对甲酰胺类化合物 88 . 该反应条件也可以适用于烷基取代的醛，并以更高的产 率得到目标产物. 在该反应条件下，直接以芳基亚胺为 底物时，反应也可以进行，说明该反应过程首先生成亚 胺中间体(Scheme 13).
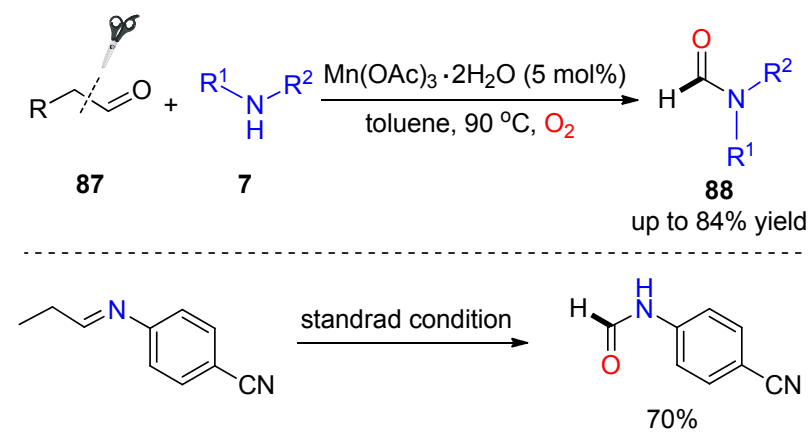

图式 13 锰催化断裂 $\mathrm{C}-\mathrm{C}$ 键醛与仲胺之间的氧插入反应 Scheme 13 Manganese catalyzed oxygenation between aldehydes and secondary amines via $\mathrm{C}-\mathrm{C}$ bond clevage

2013 年, 焦宁教题组 ${ }^{[47]}$ 通过溴化亚铜-吡啶体系催 化 1,3-二酮类化合物和一级醇之间的氧插入反应, 在氧 气环境中, 实现了芳基 $\alpha$-酮酸酯类 90 的合成. 反应机理 研究显示反应过程涉及到 $\mathrm{C}-\mathrm{C}$ 键的断裂, 同时伴随着 副产物芳基甲酸酯 91 的产生(Eq. 22). 


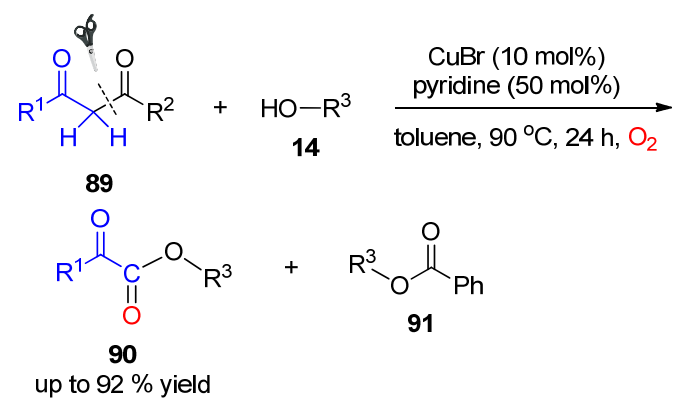

2014 年, 焦宁课题组 ${ }^{[48]}$ 通过溴化亚铜一吡啶体系进 一步催化芳基烷基酮类与一级醇之间的氧插入反应，合 成了芳基酸酯类化合物 93. 同位素标记实验显示产物 羰基中的氧约三分之二来自于酮类原料 92, 只有三分 之一的氧来自于空气中的氧. 机理显示反应同时涉及到 了 $\mathrm{C}-\mathrm{C}$ 键和 $\mathrm{O}-\mathrm{O}$ 键的断裂, 伴随着副产物醛的生成 (Eq. 23).

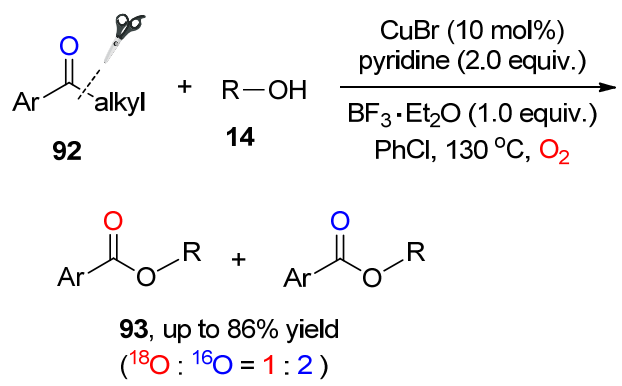

2017 年, 郭凯课题组 ${ }^{[49]}$ 报道了芳基酮与饱和的仲 胺之间的氧插入反应(Eq. 24), 该反应在异硫氰酸铜的 催化及 4- $N, N$-二甲基吡啶(DMAP)的碱性条件下, 通过 活化 $\mathrm{C}(\alpha)-\mathrm{H}$ 键进行氧气插入反应. 该反应经过芳基酮 的 $\mathrm{C}(\alpha)-\mathrm{C}(\beta)$ 键断裂、氧一氧键的断裂以及仲胺的亲核 加成, 最后铜氧气氧化得到 $\alpha$-酮酰胺类化合物. 该反应 条件下产物专一性较差, 同时会伴随着多个副产物的生 成, 例如醛类、酰胺类等副产物。

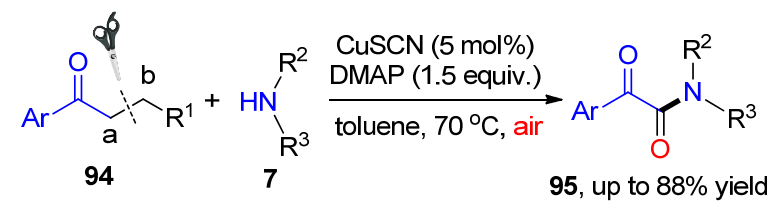

过渡金属催化断裂 $\mathrm{C}-\mathrm{C}$ 的氧插入反应目前只应用 在合成酯和酰胺类化合物, 是过渡金属催化氧气氧插入 反应的另一重要部分. 然而, 目前该类反应只涉及到 $\mathrm{C}-\mathrm{C}$ 单键的断裂, 还未涉及到断裂 $\mathrm{C}=\mathrm{C}$ 键或 $\mathrm{C} \equiv \mathrm{C}$ 键 的氧插入反应. 因此, 通过过渡金属催化断裂不饱和碳 碳键的氧插入反应是这部分未来的发展方向.

\section{3 总结与展望}

相对于传统合成含氧化合物的方法，过渡金属催化 氧气氧插入反应具有简单、高效、绿色和经济等特点. 该 类反应近几年的发展已经实现了对甲基、亚甲基、次甲 基、烯烃、炔烃、苯环、吲哚环等参与的氧插入反应，并 合成了结构多样的含羟基、羰基或含氧杂环类化合物. 目前，过渡金属催化氧气氧插入反应已经成为有机合成 或工业上合成含氧化合物的最重要的方法之一. 然而, 该类反应发展虽然非常迅速，但随着绿色化学和可持续 发展化学的新要求, 该类反应仍然存在未解决的问题和 挑战. 比如说该类反应需要多种金属参与，且反应选择 性还不够好，反应的条件比较苛刻，同时通常需要加入 催化辅助剂，底物局限性和反应立体选择性未解决等. 这些不足都限制了这类反应的广泛应用和快速发展. 近 年来, 光催化氧气氧插入反应引起了合成化学家的极大 兴趣. 通过光催化诱导自由基历程的氧插入反应避免了 过渡金属的使用，使得反应更加绿色环保. 因此，如何 实现温和条件下的氧气插入反应，如何根据该类催化体 系活性中间体设计新型自由基参与的氧插入反应及其 过渡金属催化立体选择性氧插入反应是该领域今后重 点发展的方向.

\section{References}

[1] Yin, Z.; Wang, Z.; Wu, X. F. Chin. J. Org. Chem. 2019, 39, 573 (in Chinese).

(尹志平, 王泽超, 吴小锋, 有机化学, 2019, 39, 573.)

[2] Zhang, Y.; Riemer, D.; Schilling, W.; Kollmann, J.; Das, S. ACS Catal. 2018, 8, 6659 .

[3] Thatikonda, T.; Deepake, S. K.; Das, U. Org. Lett. 2019, 21, 2523.

[4] Yuan, S.; Wang, Y.; Qiu, G.; Liu, J. B. Chin. J. Org. Chem. 2017, 37, 566 (in Chinese). (袁斯甜, 王艳华, 邱观音生, 刘晋彪, 有机化学, 2017, 37, 566.)

[5] Liang, Y. F.; Jiao, N. Acc. Chem. Res. 2017, 50, 1064

[6] Zhang, L.; Ang, G. Y.; Chiba, S. Org. Lett. 2011, 13, 1622.

[7] Wang, Y. F.; Chen, H.; Zhu, X.; Chiba, S. J. Am. Chem. Soc. 2012, 134, 11980.

[8] Zhang, C.; Xu, Z.; Zhang, L.; Jiao, N. Angew. Chem., Int. Ed. 2011, 50,11088 .

[9] Zhang, C.; Zhang, L.; Jiao, N. Adv. Synth. Catal. 2012, 354, 1293.

[10] Xu, Z.; Zhang, C.; Jiao, N. Angew. Chem., Int. Ed. 2012, 51, 11367.

[11] Huang, X.; Li, X.; Zou, M.; Pan, J.; Jiao, N. Org. Chem. Front. 2015, 2, 354

[12] Kumar, Y.; Jaiswal, Y.; Kumar, A. J. Org. Chem. 2016, 81, 12247.

[13] Xu, X.; Li, B.; Zhao, Y.; Song, Q. Org. Chem. Front. 2017, 4, 331

[14] Chen, X. L.; Peng, Y. H.; Li, Y.; Wu, M. H.; Guo, H. B.; Wang, J.; Sun, S. F. RSC $A d v$. 2017, 7, 18588.

[15] Wang, Y.; Liu, H. X.; Zhang, X. F.; Zhang, Z. L.; Huang, D. G. Org Biomol. Chem. 2017, 15, 9164.

[16] Su, Y.; Sun, X.; Wu, G.; Jiao, N. Angew. Chem., Int. Ed. 2013, 52, 9808.

[17] Sun, X.; Li, X.; Song, S.; Zhu, Y.; Liang, Y.-F.; Jiao, N. J. Am. Chem. Soc. 2015, 137, 6059.

[18] Lu, Q. Q.; Liu, Z. L.; Luo, Y.; Zhang, G. H.; Huang, Z. Y.; Wang, H. M.; Liu, C.; Miller, J. T.; Lei, A. W. Org. Lett. 2015, 17, 3402.

[19] Zhou, H.; Chen, Z. Y. Chin. J. Org. Chem. 2018, 38, 719 (in Chinese). 
(周豪, 陈知远, 有机化学, 2018, 38, 719.)

[20] Wei, W.; Liu, C. L.; Yang, D. S.; Wen, J. W.; You, J. M.; Suo, Y. R.; Wang, H. Chem. Commun. 2013, 49, 10239.

[21] Wei, W.; Wen, J. W.; Yang, D. S.; Wu, M.; You, J. M.; Wang, H. Org. Biomol. Chem. 2014, 12, 7678.

[22] Gao, W. C.; Cheng, Y. F.; Shang, Y. Z.; Chang, H. H.; Li, X.; Zhou, R.; Qiao, Y.; Wei, W. L. J. Org. Chem. 2018, 83, 11956.

[23] Toh, K. K.; Wang, Y.-F.; Ng, E. P. J.; Chiba, S. J. Am. Chem. Soc. 2011, 133, 13942.

[24] Qian, S. P.; Ma, Y. R.; Gao, S. S.; Luo, J. F. Chin. J. Org. Chem. 2018, 38, 1930 (in Chinese) (钱少平, 马尧睿, 高珊珊, 骆钧飞, 有机化学, 2018, 38, 1930.)

[25] Zhang, Y. H.; Yu, J. Q. J. Am. Chem. Soc. 2009, 131, 14654.

[26] Yan, Y.; Feng, P.; Zheng, Q.-Z.; Liang, Y.-F.; Lu, J.; Cui, Y.; Jiao, N. Angew. Chem., Int. Ed. 2013, 52, 5827.

[27] Chiba, S.; Zhang, L.; Lee, J.-Y. J. Am. Chem. Soc. 2010, 132, 7266.

[28] Tnay, Y. L.; Chen, C.; Chua, Y. Y.; Zhang, L.; Chiba, S. Org. Lett. 2012, 14, 3550 .

[29] Liang, Y.-F.; Li, X.; Wang, X.; Yan, Y.; Feng, P.; Jiao, N. ACS Catal. 2015, 5, 1956.

[30] Li, Z.; Huang, X.; Chen, F.; Zhang, C.; Wang, X.; Jiao, N. Org. Lett. 2015, 17, 584.

[31] Wu, W.; Xu, J.; Huang, S.; Su, W. Chem. Commun. 2011, 47, 9660.

[32] Bao, Y.-H.; Zhu, J.-Y.; Qin, W.-B.; Kong, Y.-B.; Chen, Z.-W.; Tang, S.-B.; Liu, L.-X. Org. Biomol. Chem. 2013, 11, 7938.

[33] Wang, C.; Zhang, L. P.; Ran, A.; Lu, P.; Wang, Y. G. Org. Lett. 2013, 15, 2982.

[34] Huang, H.; Cai, J.; Ji, X.; Xiao, F.; Chen, Y.; Deng, G. J. Angew.
Chem. 2016, 128, 315.

[35] Guo, S. H.; Wang, F.; Tao, L.; Zhang, X. Y.; Fan, X. S. J. Org. Chem. 2018, 83, 3889.

[36] Zhang, J.; Kohlbouni, S. T.; Borban, B. Org. Lett. 2019, 21, 14.

[37] Yu, L. M.; Zhong, Y.; Yu, J. C.; Gan, L.; Cai, Z. J.; Wang, R.; Jiang, X. X. Chem. Commun. 2018, 54, 2353.

[38] Yu, L. M. Ph. D. Dissertation, Sun Yat-Sen University, Guangzhou, 2018 (in Chinese). (余乐茂，博士论文，中山大学，广州, 2018.)

[39] Zhang, C.; Jiao, N. J. Am. Chem. Soc. 2010, 132, 28.

[40] Wang, J.; Wang, J.; Zhu, Y.; Lu, P.; Wang, Y. Chem. Commun. 2011, $47,3275$.

[41] Toh, K. K.; Sanjaya, S.; Sahnoun, S.; Chong, S. Y.; Chiba, S. Org. Lett. 2012, 9, 2290.

[42] Ling, F.; Li, Z. X.; Zhang, C. G.; Liu, X.; Ma, C. J. Am. Chem. Soc. 2014, 136, 10914.

[43] Ling, F.; Wan, Y.; Wang, D.; Ma, C. J. Org. Chem. 2016, 81, 2770.

[44] Liu, X.; Mao, R. J.; Ma, C. Org. Lett. 2017, 19, 6704.

[45] Pan, J.; Li, X. Y.; Qiu, X.; Luo, X.; Jiao, N. Org. Lett. 2018, 20, 2762.

[46] Zhang, C.; Xu, Z.; Shen, T.; Wu, G.; Zhang, L.; Jiao, N. Org. Lett. 2012, 14, 2362.

[47] Zhang, C.; Feng, P.; Jiao, N. J. Am. Chem. Soc. 2013, 135, 15257.

[48] Huang, X.; Li, X.; Zou, M.; Song, S.; Tang, C.; Yuan, Y.; Jiao, N. J. Am. Chem. Soc. 2014, 136, 14858.

[49] Liu, C.; Yang, Z.; Zeng, Y.; Fang, Z.; Guo, K. Org. Chem. Front. 2017, 4, 2375. 